\title{
Outcome and Complications of Laparoscopic Appendectomy in Almak Nimir University Hospital Shendi University Single Centre Experience, Sudan
}

\section{Elssayed Osman Elssayed*, Mohanned Omer Abass and Abdelrahman Babekir Mhammed}

Assistant Professor of General Surgery, Faculty of Medicine, Shendi University, Sudan

*Corresponding Author: Elssayed Osman Elssayed, Assistant Professor of General Surgery, Faculty of Medicine, Shendi University, Sudan.

Received: October 11, 2019; Published: October 24, 2019

DOI: 10.31080/ASMS.2019.03.0450

\begin{abstract}
Introduction: Appendicitis is the most common cause of abdominal pain and a prevalent reason for emergency surgery. Laparoscopic appendectomy is the method of choice, owing to its many advantages, less invasiveness, faster recovery and adequate exploration of the entire abdominal cavity than to observation in unequivocal cases. Laparoscopic surgery can serve as a diagnostic tool for patients with suspected acute appendicitis (AA), specially for female because of its low associated morbidity and mortality and it affords a direct diagnosis of the problem, allowing determination of the appropriate treatment. It is concluded that laparoscopic appendectomy should be offered as the method of choice in any patient population with suspicion of acute appendicitis. This study aim to evaluate the outcome and complications of laparoscopic appendectomies in Almak Nimir University Hospital Shendi University single centre experience.

Material and Methods: This is a prospective observational hospital base study on patients who underwent laparoscopic appendectomy for acute appendicitis from January to June 2019 in Almak Nimir University Hospital Shendi University, Sudan. 143 patients underwent laparoscopic appendectomies for acute appendicitis or suspected acute appendicitis.

Results: Out of the 143 patients analyzed, female predominant $124(86.7 \%)$ and male were 19 (13.3\%). 5 cases (3.5\%) were converted to open surgery, intraabdominal abscess encountered in 2 patients 1.4\%. Surgical-site infection was observed in 6 patients (4.2\%). The study showed statistically significant negative-positive appendectomy (normal appendix but another pathology found). Conclusions: Laparoscopic surgery can serve as a diagnostic tool for patients with suspected (AA) specially for female because of its low associated morbidity and mortality and it affords a direct diagnosis of the problem, allowing determination of the appropriate treatment. It is concluded that laparoscopic appendectomy should be offered as the method of choice in any patient population with suspicion of acute appendicitis.
\end{abstract}

Keywords: Laparoscopic Appendectomy; Open Appendectomy; Surgical Sites Infections; Almak Nimir; Shendi

\section{Introduction}

Appendicitis is the most common cause of abdominal pain and a prevalent reason for emergency surgery. The risks of developing appendicitis through lifetime is approximately $8.6 \%$ for male and $6.7 \%$ for female $[1,2]$. The incidence of (AA) is highest in the second to forth decade of life, but this condition can occur at any age [1].
Appendectomy has remained the standard approach for the treatment of most types of appendicitis $[3,4]$; however, the question of the best operative approach has attracted consistent controversy. Since 1983, after the initial description by Semm [5], laparoscopic appendectomy(LA) has been shown to offer superior benefits to open appendectomy(OA), and it has been used for various types of appendicitis. Faster recovery, reduced the rates of sur- 
Outcome and Complications of Laparoscopic Appendectomy in Almak Nimir University Hospital Shendi University Single Centre Experience, Sudan

gical site infections (SSIs), a quicker return to work, etc., have been the main advantages demonstrated by some studies [6-8].

Although the first laparoscopic appendectomy (LA) was performed in 1983, the debate continues on which surgical approach provides better management of this condition [9].

Each time, the laparoscopic approach facilitated the determination of the cause of illness without the need to modify the number or the size of trocar ports; thus, the method was a sufficient diagnostic tool in cases with pain of the lower right hypogastrium and suspected appendicitis.

This method is especially recommended for obese patients as it avoids extensive wounds that are difficult to heal after the open appendectomy (OA) [10].

Study done by Pogorelic., et al, (2019), state that, Laparoscopic appendectomy was safe and effective in children. Advantages of laparoscopic approach were shorter hospital stay, lower number of wound infections and lower usage of analgesics [11].

While surgeons are increasingly interested in minimally invasive methods, the open approach (OA) is preferred worldwide. Initially, the limitations for wider use of this technique were a consequence of surgeons' fear of intra-abdominal abscesses, longer operative time, and higher costs associated with laparoscopy [12]. However, popularization of minimally invasive techniques and access to instrumentation in a number of centers have removed economic and logistical obstacles [13]. Moreover, subsequent studies reported that operative times for the open and laparoscopic approaches are similar for experienced surgeons [12,14].

The major benefits of (LA) include potential reductions in hospitalization time, wound infections, postoperative hernias, pain level, and perioperative trauma and improvements in cosmetic results $[12,14,15]$.

Applying the rules of good laparoscopic technique, such as careful dissection, use of the tool only in the visual range, avoiding contact of the coagulation instrument's tip with intestinal serosa, irrigation only in the infected area without contamination of the entire peritoneal cavity, draining purulent focal sites, and use of drainage in cases of abundant effusions, minimizes the risk of abscesses [16].
The rate of conversion to open in the literature was 3.3\% in one study [17] to $4.4 \%$ in another study [18] and may reach up to $6.3 \%$ [19] or (14.7\%) [20].

Many studies comparing LA and OA with respect to treatment and follow-up have been conducted. These studies have reported less postoperative surgical-site infection, decreased need for analgesics, much greater visualization, rapid healing, shorter hospital stay times, and earlier return to normal activity (RTNA) rates associated with LA [21,22]. However, because of the high costs related to endostaplers, endoclips, and knottings, LA is not the most efficient operation technique $[23,24]$.

Surgical-site infection can be seen after appendectomy. Deep surgical-site infection is more common in patients who undergo LA than in patients who undergo OA. In a study on LA conducted by Ay., et al. [25], 10.7\% surgical-site infection and 7.1\% small bowel obstruction rates were reported. Another a study on LA conducted by Katsuno., et al. (2009) [26], 6.4\% surgical-site infection, 4.3\% intraabdominal abscess, and $2.1 \%$ small bowel obstruction rates were reported.

Study done in United state of America 2014, conclude that, the overall conversion rate of LA to OA was $6.3 \%$. Compared with OA, LA had a significantly lower complication rate, a lower mortality rate, a shorter mean hospital stay, and lower mean total hospital charges in both nonperforated and perforated appendices. LA has become an established procedure for appendectomy in nonperforated and perforated appendicitis in all rates exceeding OA [19].

\section{Material and Methods}

This is a prospective observational hospital base study on patients who underwent laparoscopic appendectomy for acute appendicitis from January to June 2019 in Almak Nimir University Hospital Shendi University, Sudan. The operations performed by a three surgeons, two surgeons with fellowship training in minimal invasive surgery (able to perform basic and advanced laparoscopic procedures unsupervised and manage complications; attended a formal laparoscopy course) performed a total of 103 procedures and one surgeon (able to perform basic laparoscopic procedures with minimal occasional supervision especially in the presence of complications; no formal laparoscopy course). The three-port technique was used on all patients. Prior to inserting the trocar, a Foley catheter was inserted into the bladder. A $10 \mathrm{~mm}$ trocar was then 
Outcome and Complications of Laparoscopic Appendectomy in Almak Nimir University Hospital Shendi University Single Centre Experience, Sudan

inserted under the umbilicus using the open or close technique. Pneumoperitoneum was applied with carbon dioxide (CO2), and intraabdominal pressure was fixed at 12-14 mmHg. Next, a $5 \mathrm{~mm}$ trocar was inserted into the suprapubic area before applying another $5 \mathrm{~mm}$ trocar to the left iliac fossa under direct vision. The possibility of hemorrhaging or organ injury occurring in connection with the trocar was controlled prior to performing the appendectomy. After the appendix became visible, it was lifted from the mesoappendix. A vessel sealing with monpolar diathermy was used for the mesoappendix dissection. Once the base of the appendix was revealed, it was ligated with $2 / 0$ vicryl $(10-12 \mathrm{~cm})$ using the extracorporeal knotting technique. The appendectomy specimen was then removed with a $10 \mathrm{~mm}$ trocar of the umbilicus the abdomen visualized by using zero telescope introduced through one of the $5 \mathrm{~mm}$ trocar. The specimen send for histopathology, the patients received injectable antibiotics according to guidelines. Data were analyzed using SPSS Statistical Software version 16.0.

Study area

Almak Nimir university hospital contain 300 beds located in Shendi city, which was in northern Sudan, situated on the east bank of the River nile $150 \mathrm{~km}$ northeast of Khartoum, inhabitants mainly by farmers and labors.

- Ethics approval: The study was approved by the university research committee.

\section{Results}

A total of 143 patients underwent appendectomy during the study with female predominant $124(86.7 \%)$ and male were 19 (13.3\%) (Table 1).

\begin{tabular}{|l|c|}
\hline Gender & \% \\
\hline Female & 86.7 \\
\hline Male & 13.3 \\
\hline
\end{tabular}

Table 1: Show the gender.

Second decade of life was the most common affected age group 11 - 20 years which was 79 patients $(55.2 \%)$, followed by age between 21 - 30 years old, 40 patients(28\%), while 5 patients $(3.5 \%)$ below the age of 10 years and rarely above the age of 60 just 2 patients (1.4\%) (Table 2). 66 (46.2\%) of the patient operated on had average body mass index, while 41 patients (28\%) were thin, 28 patients $(19.6 \%)$ were overweight, 8 patients were obese (5.6\%) (Table 3).

\begin{tabular}{|l|c|}
\hline Age group & $\mathbf{\%}$ \\
\hline $0-10$ & 3.5 \\
\hline $11-20$ & 55.2 \\
\hline $21-30$ & 28 \\
\hline $31-40$ & 6.3 \\
\hline $41-50$ & 4.2 \\
\hline $51-60$ & 1.4 \\
\hline $61-70$ & 1.4 \\
\hline
\end{tabular}

Table 2: Show age group.

\begin{tabular}{|l|c|}
\hline Body mass index & $\mathbf{\%}$ \\
\hline Thin & 28.7 \\
\hline Average & 46.2 \\
\hline Over weight & 19.6 \\
\hline Obese & 5.6 \\
\hline
\end{tabular}

Table 3: Show body mass index.

The majority of the patients 128 (89.5\%) required simple analgesia to alleviate postoperative pain, while 11 (7.7\%) patients did not require analgesia, 4 patients $(2.8 \%)$ had intractable pain needs opiod for pain (Table 4).

\begin{tabular}{|l|c|c|c|}
\hline Analgesia & $\mathbf{\%}$ & Complications & $\mathbf{\%}$ \\
\hline No need & 7.7 & No complications & 93.7 \\
\hline Simple analgesia & 89.5 & Conversion & 3.5 \\
\hline Opioids & 2,8 & Preperitoneal & 2.1 \\
\hline & & Bladder injury & 0.7 \\
\hline
\end{tabular}

Table 4: Show postoperative analgesia \& the intraoperative complications.

93.7\% of the patients (134) had no intraoperative complications, one patients $(0.7 \%)$ had bladder injury, while $3(2.1 \%)$ patients had preperitoneal insufflations and 5 patients $(3.5 \%)$ were converted to open approach (Table 4).

(134) of the patients (93.7) had no postoperative complications, $6(4.2 \%)$ patients had superficial wound infections and $2(1.4 \%)$ patients had intraabdominal abscess $1(0.7 \%)$ patient had postoperative internal bleeding (Figure 1). 
post operative complications

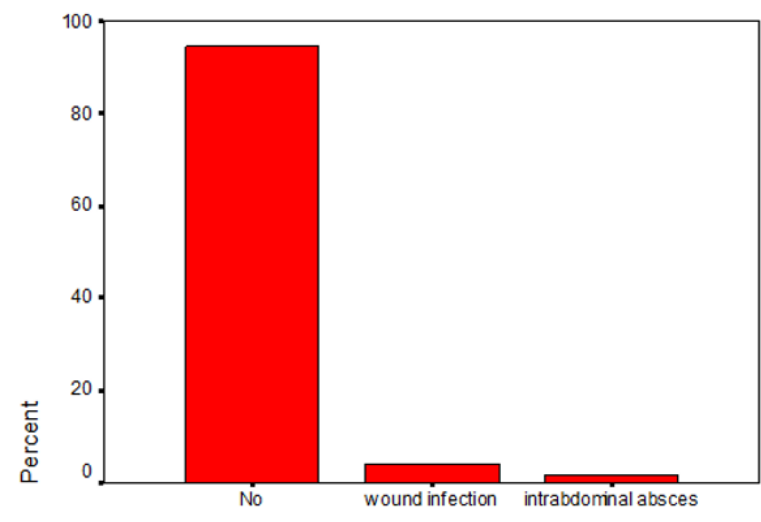

post operative complications

Figure 1: Show the postoperative complications.

128 (89.5\%) patients had inflamed appendix, while 12(8.4\%) patients had negative-positive appendectomy (normal appendix but another pathology found ) only $3(2.1 \%)$ patients had negativenegative appendectomy (normal appendix and no other pathology found) (Table 5).

\begin{tabular}{|l|c|c|c|}
\hline Status of the appendex & $\mathbf{\%}$ & Hospital stay & $\mathbf{\%}$ \\
\hline Inflammed & 89.5 & Less than 24 hour & 93 \\
\hline Negative in positive & 8.4 & $1-3$ days & 3.5 \\
\hline Negative in negative & 2,1 & More than 3 days & 3.5 \\
\hline
\end{tabular}

Table 5: Show the pathological state of the appendix and hospital stay.

Majority of the patients 133 (93\%) were stay in the hospital less than 24 hours, 5 (3.5\%) patients stay less than 3 days, while another $5(3.5 \%)$ patient stay more than 3 in the hospital (Table 5).

\section{Discussion}

This is prospective observational hospital base study done in Almak Nimir University Hospital, 143 patients were enrolled in the study, with female predominant $124(86.7 \%)$ and male were $19(13.3 \%)$, there is big variation between our study and the literature in the incidence of appendicitis between male and female $[1,2,27]$ which was due to our tendency to operate on female by laparoscopic approach, There are two issues here. The first is that diagnostic workup of clinical presentation indicative of acute appendicitis, in a certain percentage cannot exclude or confirm intraabdominal pathology. In such cases, due to unclear diagnosis, especially in women of generative age, exploration is indicated, with laparoscopy as the best approach because of two basic advantages, i.e. more detailed exploration of all four quadrants of the peritoneal cavity and less abdominal wall trauma. Secondly, patients with acute gynecologic conditions excluded whose clinical presentation indicates exploration are referred to the emergency surgeon. However, in a certain percentage, it is a gynecologic pathology.

Second decade of life was the most common affected age group (55.2\%), followed by 20 to 30 years age group finding completely consistent with the literature [1,28], Storm-Dickerson TL and his colleague state that, as world demographics trend towards an increasing number of the elderly, the absolute number of elderly requiring treatment for $\mathrm{AA}$ will also increase. Unlike in younger patients, diagnosing AA in the elderly may be challenging. In our study patient above 60 years old just 2 patients.

Concerning the body mass index, fortunately most of our patients had average weight $46.2 \%$, while $19.6 \%$ of the patients were obese and $5.6 \%$ overweight, This method is especially recommended for obese patients as it avoids extensive wounds that are difficult to heal after the open appendectomy [10].

Postoperative pain can be assessed quantitatively by the requirements for analgesics and by means of VAS scores after surgery. However, the various types of analgesics and routes of administration make it difficult to estimate the degree and relief of pain [29], in our study we assessed the pain according to the complain of the patients, The majority of the patients 128 (89.5\%) required simple analgesia in to alleviate postoperative pain, while 11 patients $(7.7 \%)$ did not require analgesia, only 4 patients $(2.8 \%)$ had intractable pain needs opiod for pain relief, finding were completely consistent with literature $[10,30,31]$. The results of our study indicate that decreased need for postoperative narcotic analgesics. In our study, fortunately $93.7 \%$ of the patients had no intraoperative complications, while 3 patients $(2.1 \%)$ had preperitoneal insufflations one patient had bladder injury during the access by verres needle and patient developed bleeding from the port site, after investigations the patient had hematological disease. 
Complicated appendicitis, for example, periappendiceal abscess, perforation, and difficult appendix stump for obvious reasons are risk factors for conversion [32,33]. 5 patients (3.5\%) were converted to open approach, 4 patients due to difficult appendix stump manipulation and one patient due to perforated appendix. It should be emphasized that conversion is usually inevitable and should not be considered an inferior treatment. In a previous analysis by Kim., et al. [34] conversion after attempted laparoscopic appendectomy was associated with a longer operative time but it did not affect postoperative morbidity and length of stay when compared with an initially open surgery [35].

Although some studies have shown that laparoscopic treatment has a higher incidence of intra-abdominal abscess [36-39], in our study intraabdominal abscess encountered in 2 patients $1.4 \%$. in some study was (12.5\%) [40]. Applying the rules of good laparoscopic technique, such as careful dissection, use of the tool only in the visual range, avoiding contact of the coagulation instrument's tip with intestinal serosa, irrigation only in the infected area without contamination of the entire peritoneal cavity, draining purulent focal sites, and use of drainage in cases of abundant effusions, minimizes the risk of abscesses [41] (figure 1).

Some authors have shown that LA is safe and leads to faster recovery with decreasing wound infection [38,42]. The infectious complications, especially SSIs and IAA, are the most common complications of appendectomy, and they are considered significant indicators of a procedure's safety [43]. Generally, it was shown that the overall incidence of SSIs was lower in LA patients [44,45], most likely because LA extracts the appendix via a bag or trocar and open appendectomy removes it directly via the wound, and the wounds in laparoscopic appendectomy are smaller than the wounds in OA [46]. Surgical-site infection was observed in 6 patients $(4.2 \%)$, which was goes with literature $(1.3 \%)$ in one study [47], 6.4\% in other study [26].

Some believe that because of laparoscopy less invasiveness and better exploration of the entire peritoneal cavity, which is impossible through McBurney's incision, laparoscopic surgeons replace observation in unclear cases with early exploration. The group submitted to early laparoscopic exploration also includes women with acute or chronic pain in the lower right quadrant, which could lead to a higher rate of negative appendectomy but also to the high rate of positive other intra-abdominal pathology [48], in our study 128 patients $(89.5 \%)$ had inflamed appendix, while 12 patients (8.4\%) had negative-positive appendectomy (normal appendix but another pathology found), mainly female patients, the other pathology was gynecological. 3 patients $(2.1 \%)$ had negative-negative appendectomy (normal appendix and no other pathology found).

Most of the studies pointing towards the advantage of laparoscopic approach was shorter hospital [11,21,22]. Our series show that majority of the patients 133 (93\%) were stay in the hospital less than 24 hours, 5 patients (3.5\%) stay less than 3 days, while other 5 patient stay more than 3 in the hospital, figure was comparable with literature.

Laparoscopic appendectomy was newly adopted advance services in our hospital, in the beginning we struggle a lot to convince the patients and co-patients with advantages and benefits of laparoscopic surgery, with time and practice the number of surgeries increases steadily, in males they wants to take the benefit of reduce the hospital stay and early return to their work specially they are farmers and in dire need for time and early return to their full activities, for females, the cosmetic benefit and the diagnostic accuracy of gynecological pathologies were both justify the switch toward laparoscopic surgery.

\section{Conclusions}

Laparoscopic appendectomy is the method of choice, owing to its many advantages, less invasiveness, faster recovery and adequate exploration of the entire abdominal cavity than to observation in unequivocal cases. Laparoscopic surgery can serve as a diagnostic tool for patients with suspected AA specially for female because of its low associated morbidity and mortality and it affords a direct diagnosis of the problem. The study showed statistically significant negative-positive appendectomy (normal appendix but another pathology found).

\section{Bibliography}

1. Addiss DG., et al. "The epidemiology of appendicitis and appendectomy in the United States". American Journal of Epidemiology 132.5 (1990): 910-925.

2. Gomes CA., et al. "Acute appendicitis: proposal of a new comprehensive grading system based on clinical, imaging and laparoscopic findings". World Journal of Emergency Surgery 10 (2015): 60. 
3. LL Rocha., et al. "Antibiotics alone versus appendectomy to treat uncomplicated acute appendicitis in adults: what do meta-analyses say?". World Journal of Emergency Surgery 10 (2015).

4. Y Tanaka., et al. "Long-term outcomes of operative versus nonoperative treatment for uncomplicated appendicitis". Journal of Pediatric Surgery 50 (2015): 1893-1897.

5. K Semm. "Endoscopic appendectomy". Endoscopy 15.2 (1983): 59-64.

6. SL Lee., et al. "Laparoscopic vs open appendectomy in children: outcomes comparison based on age, sex, and perforation status Arch". Surgery 146 (2011): 1118-1121.

7. E Southgate., et al. "Laparoscopic vs open appendectomy in older patients Arch". Surgery 147 (2012): 557-562.

8. L Enochsson., et al. "Laparoscopic vs open appendectomy in overweight patients Surg”. Endoscopy 15 (2001): 387-392.

9. Rosin D. "Value of laparoscopic appendectomy in the elderly". World Journal of Surgery 33 (2009): 923-924.

10. Varela JE., et al. "Laparoscopy should be the approach of choice for acute appendicitis in morbidly obese". The American Journal of Surgery 196 (2008): 218-222.

11. Pogorelic Z., et al. "Comparison of Open and Laparoscopic Appendectomy in Children: A 5-year Single Center Experience". Indian Pediatrics 56.4 (2019): 299-303.

12. Ortega AE., et al. "A prospective, randomized comparison of laparoscopic appendectomy with open appendectomy. Laparoscopic Appendectomy Study Group". The American Journal of Surgery 169 (1995): 208-212.

13. Mitura K., et al. "The experience and awareness of laparoendoscopic procedures among Polish surgeons in everyday clinical practice". WideochirInne Tech Maloinwazyjne 12 (2017): 13-18.

14. Milewczyk M., et al. "A prospective, randomized, unicenter study comparing laparoscopic and open treatments of acute appendicitis". Surgical Endoscopy 17 (2003): 1023-1028.

15. Pedersen AG., et al. "Randomized clinical trial of laparoscopic versus open appendicectomy". British Journal of Surgery 88 (2001): 200-205.
16. Cao J., et al. "Laparoscopic procedure is not independently associated with the development of intra-abdominal abscess after appendectomy: a multicenter cohort study with propensity score matching analysis". Surgical Laparoscopy Endoscopy and Percutaneous Techniques 27.5 (2017): 409-414.

17. Mitura Kryspin and Dorota Wyrzy kowsk. "Outcomes of Open and Laparoscopic Appendectomy with Single Endoloop Stump Closure". Journal of the Society of Laparoendoscopic Surgeons 22.4 (2018): e2018.00062.

18. Dumas RP., et al. "Laparoscopic Appendectomy: A Report on 1164 Operations at a Single-Institution, Safety-Net Hospital". The American Surgeon 284.6 (2018):1110-1116.

19. Masoomi H., et al. "Laparoscopic appendectomy trends and outcomes in the United States: data from the Nationwide Inpatient Sample (NIS), 2004-2011". The American Surgeon 80.10 (2014):1074-1077.

20. Rajab Ali., et al. "Laparoscopic Appendectomy for Acute Appendicitis: Is This a Feasible Option for Developing Countries?". Saudi Journal of Gastroenterology 16.1 (2010): 25-29.

21. Hansen JB., et al. "Laparoscopic versus open appendectomy: prospective randomized trial". World Journal of Surgery20 (1996): 17-20.

22. Heikkinen TJ., et al. "Cost-effective appendectomy: open or laparoscopic? A prospective, randomized study". Surgical Endoscopy 12 (1998): 1204-1208.

23. Hanssen A., et al. "Laparoscopic appendectomy using a polymeric clip to close the appendicular stump". Journal of the Society of Laparoendoscopic Surgeons 11 (2007): 59-62.

24. Beldi G., et al. "Analysis of stapling versus endoloops in appendiceal stump closure". British Journal of Surgery 93 (2006): 1390-1393.

25. Ay N., et al. "Comparison of outcomes of laparoscopic intracorporeal knotting technique in patients with complicated and noncomplicated acute appendicitis". Therapeutics and Clinical Risk Management 11 (2015): 1213-1216.

26. Katsuno G., et al. "Laparoscopic appendectomy for complicated appendicitis: a comparison with open appendectomy". World Journal of Surgery 33 (2009): 208-214. 
27. Hugo JR and Bonatti. "Development of a Two Port Laparoscopic Appendectomy Technique at a Rural Hospital". Minimally Invasive Surgery (2019): 9761968.

28. Buckius MT., et al. "Changing epidemiology of acute appendicitis in the United States:study period 1993-2008”. Journal of Surgical Research 175 (2015): 185-190.

29. S Yeom., et al. "Comparison of the outcomes of laparoscopic and open approaches in the treatment of periappendiceal abscess diagnosed by radiologic investigation". Journal of Laparoendoscopic and Advanced Surgical Techniques 24 (2014): 762-769.

30. Li X Zhang J., et al. "Laparoscopic versus conventional appendectomy - a metaanalysis of randomized controlled trials". BMC Gastroenterology 10 (2010): 129.

31. Sauerland S., et al. "Laparoscopic versus open surgery for suspected appendicitis". The Cochrane Database of Systematic Reviews 10 (2010): CD001546.

32. Lin YM., et al. "Laparoscopic appendectomy for complicated acute appendicitis does not result in increased surgical complications". Asian Journal of Surgery 35 (2012): 113-116.

33. Gomes CA., et al. "Lessons learned with laparoscopic management of complicated grades of acute appendicitis". Journal of Clinical Medicine Research 6 (2014): 261-266.

34. Kim JK., et al. "Does conversion adversely impact the clinical outcomes for patients with complicated appendicitis?". Journal of Laparoendoscopic and Advanced Surgical Techniques 26 (2016): 635-640.

35. Southgate E., et al. "Laparoscopic vs open appendectomy in older patients”. The Archives of Surgery 147 (2012): 557-562.

36. Horvath P., et al. "Comparison of clinical outcome of laparoscopic versus open appendectomy for complicated appendicitis". Surgical Endoscopy 31 (2017): 199-205.

37. Hanna MH., et al. "Laparoscopic right hemicolectomy: shortand long-term outcomes of intracorporeal versus extracorporeal anastomosis". Surgical Endoscopy 30 (2016): 3933-3942.

38. Taguchi Y., et al. "Laparoscopic versus open surgery for complicated appendicitis in adults: a randomized controlled trial". Surgical Endoscopy 30 (2016): 1705-1712.
39. Schlottmann F., et al. "Could an abdominal drainage be avoided in complicated acute appendicitis? Lessons learned after 1300 laparoscopic appendectomies". International Journal of Surgery 36 (2016): 40-43.

40. Tian-Chong Wu., et al. "Efficacy of emergency laparoscopic appendectomy in treating complicated appendicitis for elderly patients". Saudi Medical Journal 38.11 (2017): 1108-1111.

41. Cao J., et al. "Laparoscopic procedure is not independently associated with the development of intra-abdominal abscess after appendectomy: a multicenter cohort study with propensity score matching analysis". Surgical Laparoscopy, Endoscopy and Percutaneous Techniques 27.5 (2017):409-414.

42. Jeon BG., et al. "Prolonged operative time in laparoscopic appendectomy: Predictive factors and outcomes". International Journal of Surgery 36 (2016): 225-232.

43. G Cipe., et al. "Laparoscopic versus open appendectomy: where are we now?". Chirurgia (Bucur) 109.4 (2014): 518-522.

44. G Markides., et al. "Laparoscopic versus open appendectomy in adults with complicated appendicitis: systematic review and meta-analysis". World Journal of Surgery 34 (2010): 20262040.

45. H Masoomi., et al. "Comparison of outcomes of laparoscopic versus open appendectomy in adults: data from the nationwide inpatient sample (NIS), 2006-2008". Journal of Gastrointestinal Surgery 15 (2011): 2226-2231.

46. X Li., et al. "Laparoscopic versus conventional appendectomya meta-analysis of randomized controlled trials". BMC Gastroenterology 10 (2010): 129.

47. Mehmet Aziret., et al. "Comparison of open appendectomy and laparoscopic appendectomy with laparoscopic intracorporeal knotting and glove endobag techniques: A prospective observational study". Turkish Journal of Surgery 33.4 (2017): 258266.

48. Lee M., et al. "The morbidity of negative appendicectomy". Annals of the Royal College of Surgeons of England 96.7 (2014): 517-520.

\section{Volume 3 Issue 11 November 2019 (C) All rights are reserved by Elssayed Osman Elssayed., et al.}

